\title{
Metástasis hepática de meningioma intracraneal recurrente: reporte de caso
}

\author{
Hepatic metastasis from recurrent intracranial meningioma: case report
}

Metástase hepática de meningioma intracraniano recorrente: relato de caso

\author{
Liseth Estefanía Rubio Vega a , Stalin Roberto Villagrán Pancho ${ }^{10}$ b, Evelyn Carolina Polanco \\ Jácome (D) ${ }^{c}$, Ana Villacís ${ }^{d}$, Mayra Alejandra Bautista Ponce (iD) e, Luis Alberto Pinza Reinoso (iD $f$
}

\begin{abstract}
a Centro de Salud Shell Tipo A, Shell, Ecuador; ' Hospital General San Francisco, Quito, Ecuador; ' Servicio de Anatomía Patológica, Hospital General San Francisco, Universidad Central del Ecuador, Quito, Ecuador; d Servicio de Gastroenterología, Hospital General San Francisco, Universidad Central del Ecuador, Universidad de las Américas, Quito, Ecuador; e Universidad Central del Ecuador, Quito, Ecuador; ${ }^{\text {f Servicio }}$ de Medicina Interna, Hospital General San Francisco de Quito, Pontificia Universidad Católica del Ecuador, Universidad Central del Ecuador, Quito, Ecuador.
\end{abstract}

\section{Correspondencia a:}

Luis Alberto Pinza Reinoso, luchopinza@yahoo.com

Recibido: 29 de septiembre, 2020 Aceptado: 15 de noviembre, 2020 Publicado: 30 de diciembre, 2020

\section{CASO CLÍNICO}

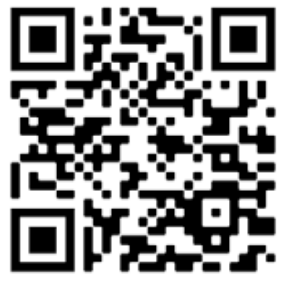

Escanea en tu dispositivo móvil o revisa este artículo en: https:// revistaclinicaguayaquil.org

\section{RESUMEN}

Los tumores cerebrales son un grupo heterogéneo de neoplasias de distintas líneas celulares. Estos pueden ser primarios o secundarios, según donde se originan, tanto en tejido del sistema nervioso central o en otro sitio del cuerpo. Los tumores primarios más frecuentes son el meningioma y el glioblastoma, mientras que las metástasis más frecuentes son en pulmón, mama y piel. Presentamos el caso de un paciente masculino de 29 años de edad con cuadro de dolor en epigastrio de 2 meses de evolución. En la tomografía computarizada simple y contrastada de abdomen se evidencian múltiples lesiones tumorales a nivel hepático las cuales se filian al antecedente de meningioma occipital extirpado hace 5 años. El meningioma atípico extracraneal de localización hepática, es una presentación inusual, razón por la cual fue importante tomarlo en cuenta al momento de evaluar los diagnósticos diferenciales de masas hepáticas.

Palabras clave: meningioma; tumor; hígado; metástasis

\section{ABSTRACT}

Brain tumors are a heterogeneous group of neoplasms of different cell lines. These can be primary or secondary, depending on where they originate, either in the central nervous system or elsewhere in the body. The most common primary tumors are meningioma and glioblastoma, while the most common metastases are in the lung, breast, and skin. We present the case of a 29-year-old male patient with history of epigastric pain of 2 months. In the simple and contrasted computed tomography of the abdomen, multiple tumor lesions were evidenced in the liver, which are thought to be related to the past medical history of the occipital meningioma excised 5 years ago. The atypical extracranial meningioma located in the liver is an unusual presentation, which is why it was important to take it into account when evaluating differential diagnoses of liver masses.

Key words: meningioma; tumor; liver; metastasis.

\section{RESUMO}

Os tumores cerebrais são um grupo heterogêneo de neoplasias de diferentes linhagens celulares. Podem ser primários ou secundários, dependendo de onde se originam, seja no tecido do sistema nervoso central ou em qualquer outra parte do corpo. Os tumores primários mais comuns são meningioma e glioblastoma, enquanto as metástases mais comuns são no pulmão, mama e pele. Apresentamos o caso de um paciente do sexo masculino, 29 anos, com dor epigástrica de dois meses de evolução. Na tomografia computadorizada de abdome simples e contrastada, evidenciam-se múltiplas lesões tumorais ao nível do fígado, as quais relaciona-se com o antecedente de meningioma occipital excisado há 5 anos. $\mathrm{O}$ 
meningioma extracraniano atípico localizado no fígado é uma apresentação incomum, porém no processo de avaliação é importante levar em consideração o diagnóstico diferencial de massas hepáticas.

Palavras-chave: meningioma; tumor; fígado; metástase

\section{INTRODUCCIÓN}

Los meningiomas son neoplasias primarias del sistema nervioso central, tienen un origen en las células meningoteliales (aracnoides). Representan el tipo más común de tumor benigno cerebral de localización extraaxial. De los meningiomas, el $90 \%$ son benignos, resultando en una baja tasa de recurrencias (7$20 \%)$ y de etiología maligna (1 - 2.8\%) (1). Las metástasis extracraneales son raras y ocurren en el $0.1 \%$ de los casos (2). Observándose metástasis principalmente a pulmón en un $60 \%$ de los casos, seguido a nivel hepático, ganglionar y hueso (2). Por estas características de tener baja recurrencia y su rara frecuencia de realizar metástasis a sitios extracraneales, presentamos el siguiente caso clínico de un paciente con dolor abdominal y antecedente quirúrgico de exéresis de meningioma en el 2015, que presenta evidencia en tomografía de masa hepática.

\section{DESCRIPCIÓN DEL CASO CLÍNICO}

Paciente masculino de 29 años de edad, con antecedente de ceguera debido a exéresis de meningioma occipital bilateral por dos ocasiones hace 5 años (Figura 1A). El paciente acudió con dolor abdominal de dos meses de evolución localizado en epigastrio e hipocondrio derecho, de intensidad moderada (6/10), acompañado de pérdida de peso.

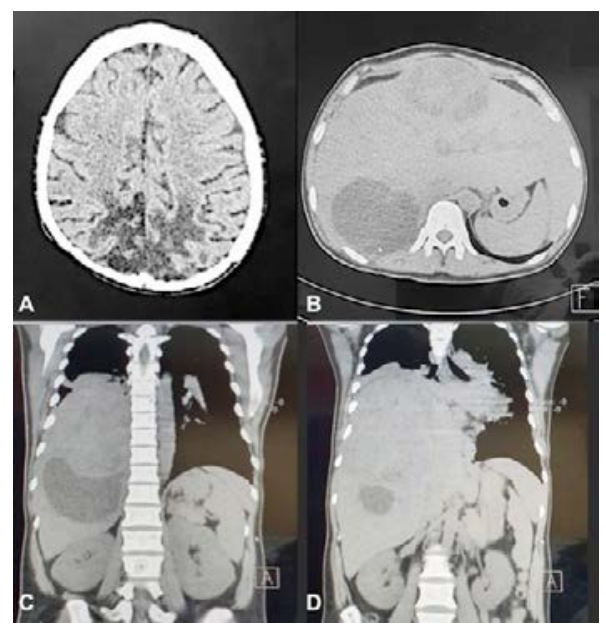

Figura 1. A. Corte axial de tomografía computarizada simple de cráneo con hipodensidad en región parietal-occipital secundario a exéresis de meningioma. B. Corte axial de tomografía computarizada simple de abdomen, se observan múltiples lesiones tumorales a nivel hepático. C. y D. Cortes coronales de tomografía computarizada simple de abdomen, se observan múltiples lesiones tumorales a nivel hepático.
Al examen físico se encontró una masa dura, móvil, no dolorosa a la palpación a nivel de epigastrio; la evaluación neurológica demostró ceguera, movilidad y sensibilidad conservadas. En la tomografía simple y contrastada de abdomen y pelvis se evidenciaron varias masas de aspecto tumoral a nivel hepático (Figuras 1B-1D); estas masas fueron dispuestas para la realización de biopsias y enviadas para estudio histopatológico. En el estudio de histopatología se evaluaron microscópicamente tres cortes de tejido blando teñidos con hematoxilina y eosina (H\&E). Las secciones microscópicas del tumor demostraron fragmentos de tejido hipercelular compuestos de células ahusadas monótonas con núcleos alargados, cromatina dispersa y citoplasma escaso. En áreas focales se observó un patrón de crecimiento en espiral (figura 2A). Basados en los hallazgos histomorfológicos, sumado al antecedente de meningioma primario, el resultado preliminar de la biopsia fue sospechoso para meningioma metastásico.

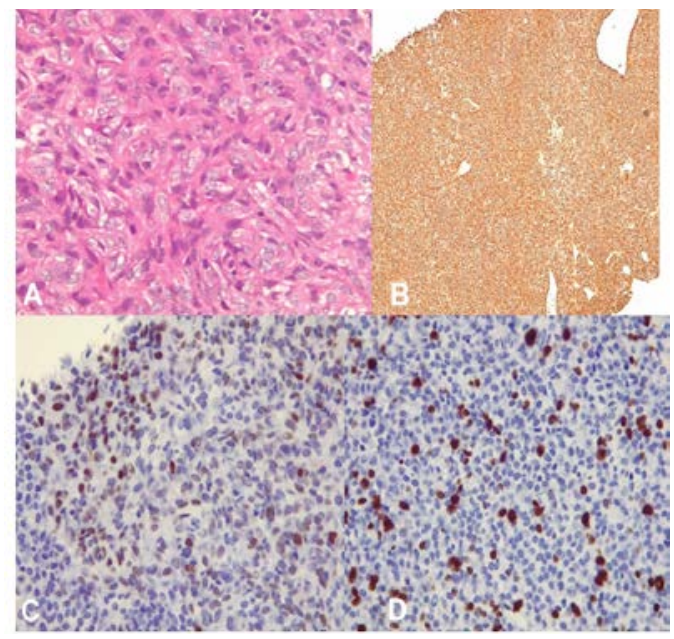

Figura 2. A. Sección microscópica, se evidencia proliferación de células ahusadas y focalmente se observa un patrón en espiral (hematoxilina y eosina H\&E, 40X). B. Estudio de inmunohistoquímica que muestra células tumorales positivas fuerte y difusamente para vimentina (10X). C. Estudio de inmunohistoquímica que muestra células tumorales positivas focalmente para receptor de progesterona (40X). D. El índice de proliferación celular Ki-67 muestra tinción en $40 \%$ de los núcleos (40X).

Los resultados de la inmunotinción revelaron un tumor fuerte y difusamente positivo (+) para vimentina, además de positividad fuerte focal para receptor de progesterona (Figuras 2B-2D). Las tinciones S-100 y EMA mostraron positividad débil focal. El índice de proliferación celular Ki67 demostró positividad en $40 \%$ de los núcleos tumorales, a diferencia las pancitoqueratinas (AE1/AE3) y HMB-45 resultaron negativos (-) en el tumor. Con estos resultados de inmunotinción el tumor fue catalogado como un meningioma metastásico de bajo grado (grado I). 


\section{DISCUSIÓN}

Los meningiomas constituyen el tumor intracraneal primario más frecuente (1520\%) y el tumor extraparenquimatoso de mayor prevalencia en el compartimento supratentorial en el adulto. En la médula se asientan aproximadamente el $10-15 \%$ de todos los meningiomas y constituyen hasta el $75 \%$ de todos los tumores intradurales a este nivel. En alrededor del $10 \%$ de los casos, los meningiomas pueden ser múltiples (3).

La incidencia de los meningiomas se incrementa con la edad y la mayoría son diagnosticados en los adultos, con un aumento significativo a partir de los 65 años de edad. Existe un predominio en el sexo femenino (relación femenino:masculino $>2: 1$ ) y en la raza afro descendiente (4). El $98.6 \%$ de los meningiomas diagnosticados son benignos (grado I); el resto son meningiomas atípicos (grado II) y mucho más infrecuente son los malignos o anaplásicos (grado III) (5). El paciente presentaba un tumor de características histopatológicas de meningioma grado I.

La incidencia de los meningiomas extracraneales es muy baja, representando el $0.1 \%$ de los casos (2). Se han descrito dos patrones de diseminación diferentes: hematógenos o linfáticos; cuando ocurre diseminación extraneural, los meningiomas tienden a metastatizar con mayor frecuencia a los pulmones, seguidos por el hígado, los ganglios linfáticos y el hueso (6).

Según Kessler R., et al., en su reporte de seis casos clínicos, se evidenciaron lesiones extracraneales distribuidas de la siguiente manera: dos a nivel pulmonar, dos mediastino-pleurales, uno espinal-hepático y uno hepático, presentando estos dos últimos casos, sintomatología de dolor abdominal y pérdida de peso similar al caso presentado (2). Con relación a la presencia de metástasis posterior a la resección y quimioterapia, Kessler et al., han evidenciado la recurrencia de lesiones metastásicas hasta 3 a 4 años posterior al tratamiento inicial (2).

En escasas publicaciones realizadas como la mencionada por Robbin Thomas, los tumores extracraneales de tipo metástasis, especialmente por meningioma anaplásico, representan el 2-3 \% (5). En el estudio realizado por Dalle y Magill, de 1193 pacientes, 28 de ellos presentaron metástasis extracraneales, de los cuales 1 paciente fue grado I, 16 pacientes grado II y 11 pacientes grado III (6). Reafirmando que el meningioma con metástasis extracraneal de afectación hepática con características histopatológicas de grado I, son de baja incidencia.

Por estudios de inmunotinción, los meningiomas son habitualmente positivos para receptor de progesterona y vimentina, mientras que negativos para citoqueratina y S-100 (7). Es recomendable realizar estudios genéticos y moleculares para correlacionar los hallazgos con otros tipos de tumores.

Como conclusión, en el estudio de lesiones hepáticas múltiples, el primer diagnóstico diferencial en mente debe ser lesiones por metástasis, si existe el antecedente clínico de tumores primarios. En el caso que se presentó existió diagnóstico previo de meningioma occipital de bajo grado (I), $y$, de acuerdo a la literatura, las metástasis de este tipo de meningiomas son raras y ocurren con mayor frecuencia después de varias recurrencias del tumor primario.

\section{REFERENCIAS BIBLIOGRÁFICAS}

1. Norden A, Drappatz J, Wen P. Targeted drug therapy for meningiomas. Neurosurg Focus. 2007 Feb 1;23(4):E12.

2. Kessler RA, Garzon-Muvdi T, Yang W, Weingart J, Olivi A, Huang J, et al. Metastatic Atypical and Anaplastic Meningioma: A Case Series and Review of the Literature. World Neurosurg. 2017 May;101:47-56.

3. Sanchez Rubio N, Redondo Buil P, Sal de Rellan Arango S, Santamarta Liebana E, Saiz Ayala A, Martínez Meilán M. Meningiomas típicos, atípicos y pitfalls [Internet]. Oviedo; 2012. Disponible en: https://epos.myesr.org/poster/ esr/seram2012/S-0713

4. Casas I, Báez A, Banfi N, Blumenkrantz Y, Halfon MJ, Barros $\mathrm{M}$, et al. Meningiomas en neurooncología. Neurol Argentina. 2016;8(3):210-26.

5. Thomas RZ, Dalal I. Extracranial metastases of anaplastic meningioma. BJR case reports [Internet]. 2017 Feb 7;3(2):20150092. Disponible en: https://pubmed.ncbi.nlm.nih.gov/30363256

6. Ore CLD, Magill ST, Yen AJ, Shahin MN, Lee DS, Lucas C-HG, et al. Meningioma metastases: incidence and proposed screening paradigm. J Neurosurg JNS [Internet]. 2019;132(5):1447-55. Disponible en: https://thejns.org/view/journals/jneurosurg/132/5/article-p1447.xml

7. Senra Armas L, Andara Ramírez M, Noa Pedroso G. Metástasis hepática como forma de presentación de cáncer. Rev Cubana Med. 2015;54(1):27-39. 


\section{ACERCA DE LOS AUTORES}

1. Liseth Estefanía Rubio Vega: Médica rural en el Centro de Salud Shell Tipo A, Shell, Ecuador.

2. Stalin Roberto Villagrán Pancho: Médico General, Instituto Ecuatoriano de Seguridad Social, Hospital San Francisco, Quito, Ecuador.

ORCID: 0000-0001-947CC9782

3. Evelyn Carolina Polanco Jácome: Médico Especialista en Anatomía Patológica, Hospital San Francisco, Quito, Ecuador. Profesora de la Universidad Central del Ecuador, Facultad de Ciencias Médicas, Instituto Superior de Investigación y Posgrado, Posgrado de Anatomía Patológica, Quito, Ecuador. Médico Subespecialista en Citopatología y Patología Quirúrgica Selectiva, Long Island Jewish Hospital/Northwell, New York, Estados Unidos. Médico Especialista en Patología Anatómica y Clínica, Medical College of Wisconsin Affiliated Hospitals, Milwaukee, Estados Unidos. Médico de la Universidad Central del Ecuador, Quito, Ecuador.

ORCID: 0000-0002-3023-3070

4. Ana Villacís: Médico tratante del Servicio de Gastroenterología, Hospital General San Francisco, Quito, Ecuador. Docente de Pregrado Escuela de Medicina, Universidad de las Américas. Docente de Posgrado Escuela de Medicina, Universidad Central del Ecuador. Miembro de la Sociedad Latinoamericana para Estudio de las Enfermedades del Hígado. Miembro de la Sociedad Ecuatoriana de Gastroenterología.

5. Mayra Alejandra Bautista Ponce: Médico Posgradista en Anatomía Patológica de la Universidad Central del Ecuador, Quito, Ecuador. ORCID: 0000-0002-7068-5047

6. Luis Alberto Pinza Reinoso: Médico internista tratante del Hospital General San Francisco, IESS, Quito, Ecuador. Docente de Pregrado de la Pontificia Universidad Católica del Ecuador. Universidad Central del Ecuador.

ORCID: 0000-0002-5322-7597 